\title{
A Strategy for Multi-Agent Based Wireless Sensor Network Optimization
}

\author{
Ahmad Sardouk, Rana Rahim-Amoud, Leïla Merghem-Boulahia, \\ and Dominique Gaïti \\ ICD/ERA, FRE CNRS 2848, Université de Technologie de Troyes, \\ 12 rue Marie Curie, 10010 Troyes Cedex, France \\ \{ahmad.sardouk, rana.amoud, leila.boulahia, dominique.gaiti\}@utt.fr
}

\begin{abstract}
The multi-agent approach has been proposed in the literature as a solution for data gathering, and routing in Wireless Sensor Networks (WSNs). In these propositions, the knowledge of an agent is generally limited to a single parameter such as the energy of the sensor node and/or to the address of its next hop in a routing protocol proposition. In this paper, we propose a strategy for the agent to make a more appropriate decision to cooperate or not in a data gathering session. This strategy uses, in addition to the energy of the node, several parameters from the local view of the agent as the position of the node within the network, the network density, and the information importance degree. Through successive simulations, this strategy has proved its ability to manage cleverly the power consumption of the sensor nodes and hence to extend the WSN life time.
\end{abstract}

Keywords: Wireless sensor networks, Multi-agent systems, Energy-efficiency, Autonomic sensor network.

\section{Introduction}

The rapid technological progress in wireless sensor networks is attracting more and more applications. This domain was known by its importance for military applications where sensors are scattered behind the enemy lines to collect information. However, nowadays the WSN is proposed for medical use to monitor the patients with special diseases and prevent them from spreading. Many other applications, such as tracking and large scale environmental monitoring, require this improvement in WSN.

However, the sensor nodes have limited power defined by their batteries. The radio entity of the sensor node drains this battery in transmission, reception and even in idle state. Hardware advances in communication and battery technology will lead in overcoming some of the power problems. Thus, the constructors of sensor nodes are giving a special interest to the development of processing and memory capacity of sensor nodes. An example of this development could be seen in SunSpot [10] sensor nodes. They offer new sensor nodes with $180 \mathrm{MHz}$ of processing, 512KB of RAM and 4MB of memory with an implemented java virtual machine. However, problems will not be completely resolved and software improvements are still needed. Indeed, a sensor 
node should be able to manage its battery, to judge the importance of the gathered information, to reduce the communication of non useful or non important information, etc. In other terms, a sensor node should be autonomous and should be able to make decisions while taking into consideration its state and the state of its environment.

In this context, the multi-agent systems (MASs) emerged as an important tool to build autonomic networks. An MAS is composed of a set of agents able to cooperate with each other to exchange information and execute tasks in order to achieve a global objective.

In one of our previous papers [8], we have proposed an energy-efficient communication architecture based on the multi-agent approach. In the proposed architecture, only an agent having important information cooperates with its neighbours in order to create a data gathering session. This session summarizes the data of multiple sensor nodes into one message allowing to significantly saving energy.

In order to manage more cleverly the power consumption of the nodes, in this paper, we enhance the architecture defined in [8] by proposing an agent strategy which is a step towards the autonomic WSN. By using this strategy, the agent, implemented in a sensor node, will take into consideration several parameters before deciding to cooperate or not in a data gathering session. These parameters are obtained from the agent environment or by exchanging information with its neighbours. In this paper we define the following four parameters: (1) The energy of the node, (2) the position of the node within the network, (3) the network density in the coverage zone of the node, and (4) the importance degree of the gathered information. These parameters will be explained and discussed in details in section 4.

The rest of this paper is organized as follows. In section 2, we present briefly the multi-agent systems. In section 3, we review the agent-based solutions proposed in the literature. Next, section 4 contains the main contribution of this paper: the agent strategy. Simulations setup and results analysis are discussed in section 5 and 6 respectively. Finally, section 7 concludes the paper and introduces the future work.

\section{Multi-Agent Systems}

According to [2], an agent is a physical (robot) or virtual (real time embedded software) entity having trends and resources, able to perceive its environment, to act on it and to acquire a partial representation of it (called the local view of the agent). An agent is also able to communicate with other peers and devices and has a behaviour that fits its objectives according to its knowledge and capabilities. Furthermore, an agent can learn, plans future tasks and is able to react and to change its behaviour according to the changes in its environment.

An MAS is a group of agents able to interact and to cooperate in order to reach a specific objective. Agents are characterized by their properties that determine their capabilities. Different properties are defined like autonomy, proactive-ness, flexibility, adaptability, ability to collaborate and to coordinate tasks, mobility, etc.

According to its role within its environment, the agent acquires some of these properties. Multi-agent approach is well suited to control distributed systems. WSN are good examples of such distributed systems. This explains partly the considerable contribution of agent technology when introduced in this area. 


\section{Agent-Based Propositions in WSN}

Agents and MAS are two interesting concepts for a great number of researchers in different domains. In WSN, researchers have been interested in these two concepts in order to propose energy-efficient approaches especially in routing and data gathering problems. In this section, we present some of the proposed solutions and we underline the local view of the agent in each proposition. In fact, the decisions of an agent are based on its local view. This view presents the information of its current sensor node information as the remaining power in the node, the list of its neighbours, etc.

Since WSN is limited in energy, designing an energy-efficient routing protocol appears as a key point to extend the network life time. Therefore, authors of [5] propose an intelligent agent routing scheme over WSN. This scheme is based on building an abstract tree structure where the sink is the root. When an event happens, the information can be passed back to the sink through the tree structure. The authors propose to introduce an agent at each level of the tree. This agent transfers the information of a lower level to an upper level. At each level of the tree, they select the closet (in term of distance) sensor node to the upper level to implement the agent in this proposition, the local view of the agent is limited to the address of its upper level node and the distance to it (deduced from the received power).

Authors of [3] propose another approach based on the use of mobile agent. They propose to carry each data packet sent from a source node by an autonomous mobile agent. This agent is responsible for making the appropriate route decisions, in an energy-efficient manner, based on its local view. The authors define a forwarding table in each sensor node. This table contains the list of possible next hops with their remained power and estimated power (or cost). The estimated power is the transmission power needed to reach the next hop. In this proposition, the energy of the next hop appears as the main information in the local view of the agent.

In mobile agent propositions [1] [4], authors propose to send the processing code to the sink. This code is a part of a message called mobile agent, which contains also the list of source nodes. The mobile agent passes through each of the source nodes defined in the list, processes their data locally and concatenates them into the data field of the message. This technique provides a gain in power by (1) eliminating the local redundant information as the mobile agent processes data locally, and (2) concatenating multiple nodes information into one message, which means one message overhead for multiple source nodes information. The authors suppose a fixed list of source nodes in each mobile agent. The agent, thus, will just focus on finding its route to process and concatenate the data from its list of source nodes. Hence, the local view of the agent, when it arrives to a node, will be restricted to the local raw data and the address of the next sensor node (next source node in the list).

In our previous work [7] [8], we have proposed an information importance based communication architecture (IBC). Thus, the data gathering session starts when an agent (sensor node) detects important information. This agent invites its one hop neighbours to cooperate in order to gather the maximum possible of information and to create a cooperation message summarizing these collected information. However, the neighbour agent, who is at the same time the first hop on the path to the sink for 
the agent in question (source node), will not response the cooperation request. Indeed, once the cooperation message is ready, this neighbour agent (called intermediate agent) will receive the message and will invite its one hop neighbours' agents to cooperate. The intermediate agent will gather the information of its one-hop neighbours and extend the initial cooperating message. This message will be then sent to the next intermediate agent. The new intermediate agent will, in its turn, repeat the same scenario. This scenario will be repeated until reaching the sink node.

The example in Fig. 1 illustrates the IBC approach where we can see that there is a static agent (smile face) implemented in each node. We suppose that agent A has important information, hence it cooperates with its one hop agent neighbours ( $\mathrm{E}, \mathrm{I}$ and B) to gather their information. Then, it sends the resulting cooperation message to its first hop on the path to the sink, which is agent B. B, C, and D are the intermediate agents, each one of them cooperates with its one hop neighbours and concatenates data into the main cooperation message. We would like to underline here that the neighbour agents decide to cooperate or not following the importance of their gathered information. In this proposition, the local view of an agent was limited to the importance of the gathered information.

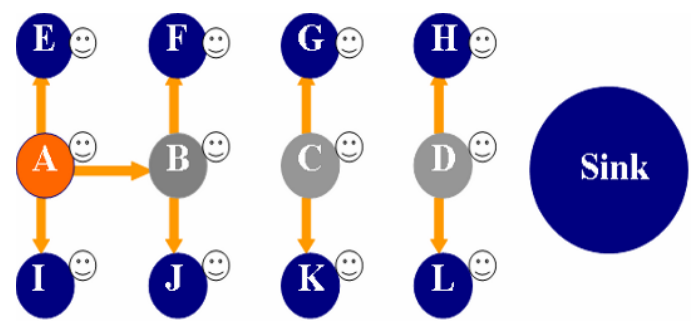

Fig. 1. Agents based communication example

The authors of [6] propose the use of agents for a power management in wireless sensor network. Unfortunately this work does not give results; however, it studies the possibility and importance of using the agents in WSN. The authors examine some of the potential decisions an agent can take regarding intelligent power management. They give the example of a node, in the middle of the network, which routes further nodes messages if it has a sufficient battery level. This work emphasizes the importance of using different characteristics of agents in WSN.

The approaches presented in this section propose the use of MASs in WSN in order to decrease the power consumption. However, in each proposition, only a few number of parameters of the agent local view have been considered by the agents in order to make decisions. In the majority of these propositions, the authors have mainly focused on the available power. However, some other parameters would be important for the longevity of a WSN such as the position of the node, the network density, etc.

In the next section, we detail our agent strategy and the parameters that it takes into account in order to make appropriate decisions. 


\section{Agent Strategy}

In the literature, an agent is generally selfish which means that it cooperates with its neighbour agents when it can and needs. This feature appears to be very important in WSNs as an agent cooperates only if there is a gain. The gain is a relative point that depends mainly, in our domain, on the sensor node life time and the relevance of cooperating with other agents. In the present work, we propose a strategy for the agent to compute the relevance of cooperation. For this strategy, we define the main parameters that may influence the relevance of the participation in cooperation. These parameters are as follow:

- $\quad$ The energy $(\mathrm{E})$;

- $\quad$ The network density (D);

- $\quad$ The position of a sensor node within the network $(\mathrm{P})$;

- The information importance (I).

We will like to note also that the relevance $(\mathrm{R})$ will be computed with an approximation of a constant (C). In addition, each one of the defined parameters has its importance factor or its priority. We express the defined parameters by the equation (1) to compute the relevance of cooperation.

$$
R=E \times \alpha+\frac{1}{D} \times \beta+\frac{1}{P} \times \theta+I \times \omega+C
$$

Where $\alpha, \beta, \theta$, and $\omega$ are the importance factor for the energy (E), the density (D), the position $(\mathrm{P})$, and the information importance degree (I) respectively.

\subsection{Energy}

The energy is an important parameter in a resource limited network such as the WSN. It is generally seen as the most important parameter. Indeed, the remaining battery level appears to be the most important thing in this parameter but it is not the only one. In order to better use the energy of a node, we define an administrator power strategy (APS) parameter. This parameter allows the network administrator to extend its WSN life time. By multiplying the available battery level by a percentage, the agent will reject some cooperation requests that it would be accepted if the administrator strategy was disabled. This rejection allows sensor nodes to save more energy, hence it extends the whole network life time.

Otherwise, the importance of the administrator power strategy could be emphasized also in the case of multi-application sensor network where the administrator will be able to define the importance of each application. Hence, the value of $(\mathrm{E})$, is given by equation (2):

$$
E=A \times A P S
$$

Where $\mathrm{E}$ is the energy parameter in equation (1), $\mathrm{A}$ is the available or remained power in the battery and APS is the administrator power strategy parameter, which is a percentage defined by the administrator depending on the application. As presented in 
the equation (2), the energy E could be just the remaining battery level if no administrator power strategy has been defined $(\mathrm{APS}=1)$. In addition, lower is the APS longer is the life time of the WSN.

\subsection{Network Density}

The network density varies from a deployment to another one and from a node to another one within the same deployment depending on the node distribution. This parameter appears as an important parameter to take into consideration. Indeed, more an agent has neighbours, less is the importance of its participation in a cooperation; that is why in the equation (1), we take the inverse of the density (D). To illustrate the importance of this parameter, let us take the example of a tracking application where the position of the desired object can be defined by at least three agents. Finding these three agents in a dense network is an easy task. However, if ten agents participate in this task, in place of three, we will have an undesired loss of power and time.

The density in this case is a relative parameter computed by each agent. There are two main reasons behind that: the first one is that each agent has a local view and there is no agent with a global network view. The second reason, which is more important, appears in the fact that for a specific task, we need cooperation between the agents of the local task zone and not farther agents. For simplicity's sake, we propose the following equation (3) to compute this density (D).

$$
D=\frac{N_{\text {real }}}{N_{\text {theoretical }}}
$$

Where $\mathrm{N}_{\text {theoretical }}$ is the theoretical number of nodes and it is given from the ideal distribution of the nodes or the grid distribution (see Fig. 2(a)) $\mathrm{N}_{\text {theoretical }}$ corresponds to the number of nodes within the radio range of a reference node $(\mathrm{RN})$. The $\mathrm{RN}$ is a node in the centre of the area to eliminate the special cases of border nodes.

$\mathrm{N}_{\text {real }}$ is the real number of neighbour nodes, which means the number of its one hop neighbour nodes appearing on its MAC layer. It should be equal, in the ideal case, to all the neighbor nodes within the radio range of the node. In Fig. 2(b), we show an example of randomly distributed nodes to give an idea about real network densities.

\subsection{Position within the Network}

The third parameter is the position of the agent node $(\mathrm{P})$ in the network. We define three types of node positions: (1) normal, (2) edge and (3) critical. The normal position is the position inside the network where the node has multiple neighbours. The edge node is a node in the border of the network and/or having a view of the network limited to one and only one neighbour. A node is considered in a critical position if it connects two parts of the network. That means, if the node runs out of battery, it may divide the network or multiple nodes behind it will become unreachable and in the best case they will require a longer route to communicate their data to the sink. This longer route is expensive in term of energy as the number of hops is increased. Fig. 2(b) presents a random deployment of 100 nodes and we have marked some of the nodes that are in critical position. 
The strategy should allow an agent in a critical position to decrease its power consumption to maintain the maximum possible the connection between the two parts of the network. Thus, the value of the importance factor of this parameter should be equal or higher than the energy or the information importance degree factor.

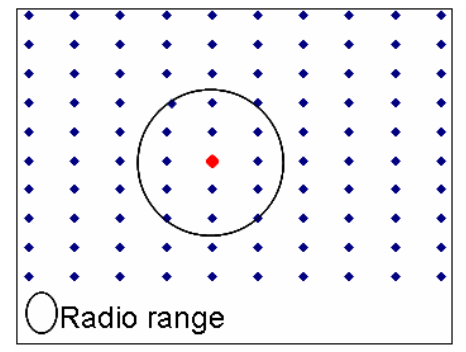

(a) Ideal distribution

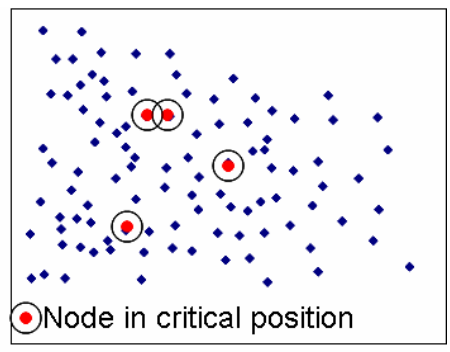

(b) Random distribution

Fig. 2. Network topology

\subsection{Information Importance Degree}

The last parameter (I) is the information importance degree that depends heavily on the desired application. This parameter could be computed by a local processing in the node. This processing allows the agent to estimate the importance of the gathered information.

For example, in a tracking application, if the detected object is the desired one or in the case of a visual application if the captured picture contains an animal face (supposing we are searching for new species in a forest), the agent will judge this information as important.

In other domains as in environmental monitoring (Humidity, temperature, etc.), the agent saves the last gathered information to compare it with the new gathered one. If the difference between the two is greater than a predefined threshold, this information will be considered as important. Conversely, the agent drops the old information and saves the recent one and marks the information as unimportant. The same technique could be used also in tracking when the object stays in the sensor zone during two or more gathering cycles.

\section{Simulation Setup}

To evaluate the relevance of our proposed agent strategy, we have carried out a set of simulation tests. These simulations compare the performance of our previous work [8] (IBC) where no agent strategy has been used, with a Strategy Based Communication (SBC), which is a communication based on our agent strategy. The IBC, presented in section 3 , is a communication architecture based on the importance of the information. We have implemented these two approaches on GlomoSim [11] which is a scalable simulation environment for wireless and wired network systems. 
In our simulation setup, as presented in Table 1, we summarize the different simulation parameters that we have used during the evaluation of our proposition. We have run our simulation over a $1000 \mathrm{mx} 1000 \mathrm{~m}$ square with a random distribution of nodes during 1000 seconds. We have limited the radio range and the data rate of each node to $87 \mathrm{~m}$ and $1 \mathrm{Mbps}$ respectively as suggested in [9]. The transmission and reception powers' parameters, which influence directly the radio range, have been chosen carefully from the ranges defined in the sun SPOT system technical document [10].

In order to test the scalability of the agent strategy and its relevance across different network densities, the simulations are done for a number of nodes varying from 100 to 900 nodes with an interval of 200 .

The local processing time is inspired from the work realized in [1] ${ }^{1}$, where the processing code is put in a message sent by the sink. This message is the mobile agent. Indeed, transferring this code from the message to the node and placing it in the appropriate place of the memory will take some time. We have estimated this time to $10 \mathrm{~ms}$. The authors of [1] have fixed the processing time to $50 \mathrm{~ms}$ which means that $40 \mathrm{~ms}$ will be sufficient in our proposition as a local processing time.

Table 1. Basic simulation parameters

\begin{tabular}{ll}
\hline Simulation Parameters & \multicolumn{1}{c}{ Values } \\
\hline Network size & $1000 \mathrm{mx} 1000 \mathrm{~m}$ \\
Node distribution & Random \\
Radio range & $87 \mathrm{~m}$ \\
Throughput & $1 \mathrm{Mbps}$ \\
Size of sensed data & 24 byte per node \\
Sensed Data Interval & 10 seconds \\
Simulation time & 1000 seconds \\
Local processing time & $40 \mathrm{~ms}$ \\
\hline
\end{tabular}

Table 2. Agent strategy equation parameters

\begin{tabular}{ll}
\hline \multicolumn{1}{c}{ Agent Strategy Parameters } & \multicolumn{1}{c}{ Values } \\
\hline Threshold of R & 0.7 \\
$\alpha, \theta, \omega$ & 0.25 \\
$\beta$ & 0.1 \\
$\mathrm{C}$ & Random $[0,0.15]$ \\
\hline
\end{tabular}

The agent strategy parameters are presented separately in section 4 . The importance factors of these parameters $\alpha, \beta, \theta$, and $\omega$ are fixed to $0.25,0.10,0.25,0.25$ respectively. The constant $\mathrm{C}$ is a random variable between 0 and 0.15 . These values reflect the importance of their correspondent parameters. By giving the same value to $\alpha, \theta$, and $\omega$, we give the same importance to the energy, position and information importance degree, in the calculation of the relevance value. The density has been given a lower priority compared to them as we suppose that it does not influence

\footnotetext{
${ }^{1}$ This work is presented in section 3 .
} 
directly the performance of the whole WSN. Based on these factors and through several simulations, we have found that the majority of cooperation relevancies $(\mathrm{R})$ were between 0.6 and 0.8 . Thus, we have chosen to fix threshold of $\mathrm{R}$ to 0.7 . These parameters are resumed in Table 2.

\subsection{Power Consumption Computation Model}

The power consumption is a main performance criterion in our work. It represents the average value of energy consumed by each sensor node during the simulation to transmit, receive, and process the data. For the transmission and reception, we use the equation (4), defined in [9]. $\mathrm{E}_{\mathrm{TX}}$ is the power consumed during transmission and $\mathrm{E}_{\mathrm{RX}}$ is the power consumed during the reception. Both of them are computed following the data length and distance of transmission (radio range of the node) $(\mathrm{l}, \mathrm{d})$ :

$$
\begin{aligned}
& E_{T X}(l, d)=l E c+l e d^{s} \\
& E_{R X}(l, d)=l E_{c}
\end{aligned} \text { where e }=\left\{\begin{array}{lll}
\mathrm{e}_{1} & \mathrm{~s}=2, & \mathrm{~d}<\mathrm{d}_{\mathrm{cr}} \\
\mathrm{e}_{2} & \mathrm{~s}=4, & \mathrm{~d}>\mathrm{d}_{\mathrm{cr}}
\end{array}\right.
$$

Where $E_{c}$ is the base energy required to run the transmitter or receiver circuitry. A typical value of $E_{c}$ is $50 \mathrm{~nJ} / \mathrm{bit}$ for a 1-Mbps transceiver; $\mathrm{d}_{\mathrm{cr}}$ is the crossover distance, and its typical value is $86.2 \mathrm{~m}$; $\mathrm{e}_{1}$ (or $\left.\mathrm{e}_{2}\right)$ is the unit energy required for the transmitter amplifier when $\mathrm{d}<\mathrm{d}_{\mathrm{cr}}$ ( or $\mathrm{d}>\mathrm{d}_{\mathrm{cr}}$ ). Typical values of $\mathrm{e}_{1}$ and $\mathrm{e}_{2}$ are $10 \mathrm{pJ} / \mathrm{bit}^{2}$ and $0.0013 \mathrm{pJ} / \mathrm{bit} \mathrm{m}^{4}$ respectively.

For the local processing consumption, we use the rules defined in [9]. The authors evaluate this energy based on the number of instructions and the frequency of the processor. In IBC and SBC, we use the processor defined in the sun SPOT technical document [10], which sets the processor frequency of their sensor nodes to $180 \mathrm{Mhz}$. According to [9], a processor with such frequency consumes approximately $0.8 \mathrm{~nJ}$ per instruction.

\section{Results and Analysis}

In this section, we present the simulation results to highlight the performance of our proposition. We show the advantages of the proposed agent strategy scheme by comparing IBC to SBC (which is an amelioration of IBC). It is important to bear in mind that in SBC we base our agent strategy only on the information importance.

We focus mainly on the efficiency of our proposition in terms of power consumption and scalability in different network densities. As presented in the simulation setup section, we have varied the number of nodes from 100 to 900 .

In Fig. 3, we plot the average power consumption per node in IBC and SBC. The results show that SBC decreases the power consumption comparing to IBC. In addition, it is clear that the saved power is more important for the higher number of nodes. These results prove that our agent strategy is significantly better designed for scalable or dense networks than the IBC approach. Indeed, for a number of nodes varying from 100 till 900, the power consumption obtained by using the agent strategy is in average reduced by a factor of 1.5 which means an important amount of saved power. 


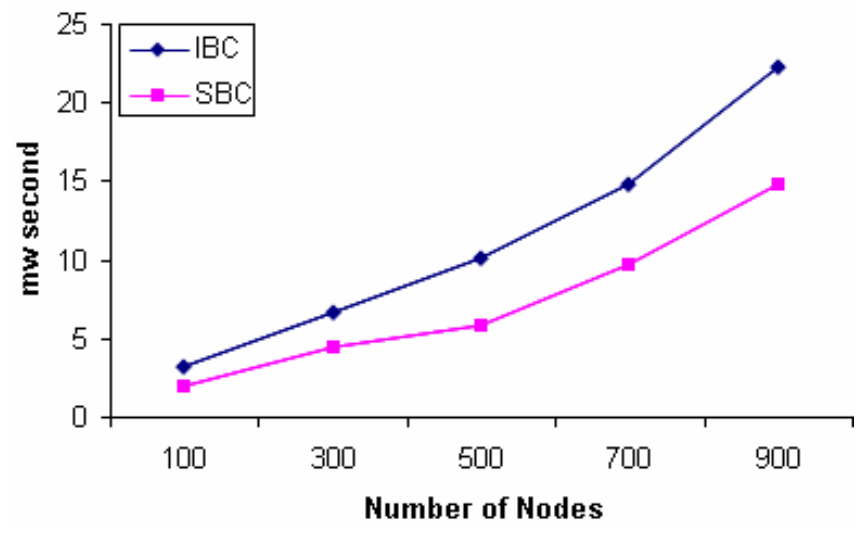

Fig. 3. Average power consumption per node

To show the effects of the administrator power strategy (APS) on the life time of the WSN, we ran a set of simulations with four different values of APS: $25 \%, 50 \%$, $75 \%$, and $100 \%$. These percentages, as presented in section 3, define for the agent the amount of energy that it can use to evaluate the relevance of cooperation.

Fig. 4 shows that when APS is equal to $75 \%$ (Fig. 4), the power consumption per node is divided by two approximately comparing to APS $=100 \%$ (no strategy). Hence, the network life time has been multiplied by two approximately.

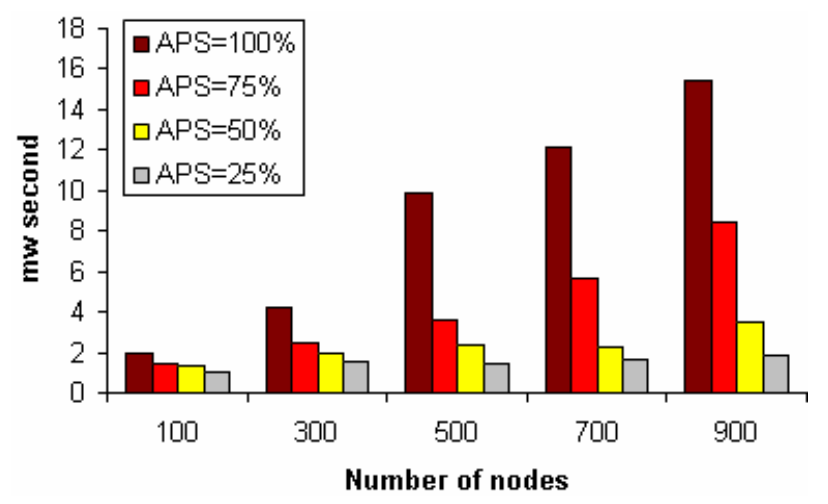

Fig. 4. Average power consumption per node for different administrator strategies

In addition, the results confirm that lower is the APS longer is the life time of the WSN. We can also observe that the network scale and density do not degrade the optimization of the network life time. The definition of this parameter depends highly on the type of the application and the WSN administrator strategy.

Fig. 5 compares the power consumption per node for the nodes in critical positions in both approaches. As we can observe, the agent strategy decreases the average power consumption of these nodes in an important manner. It shows also that more the network is dense more the amount of decreased power is important. We can observe also, that for 700 nodes, the agent strategy divided by two the consumption of these nodes 
and for 900 nodes, this optimization remained important where the agent strategy divides the consumption by more than 1.5. In addition, in a non dense network, the power consumption has been divided by a factor varying from 1.5 to 2 . Hence, we can deduce from these curves that the agent strategy offers a better power management for nodes in critical positions independently from the network scale and density.

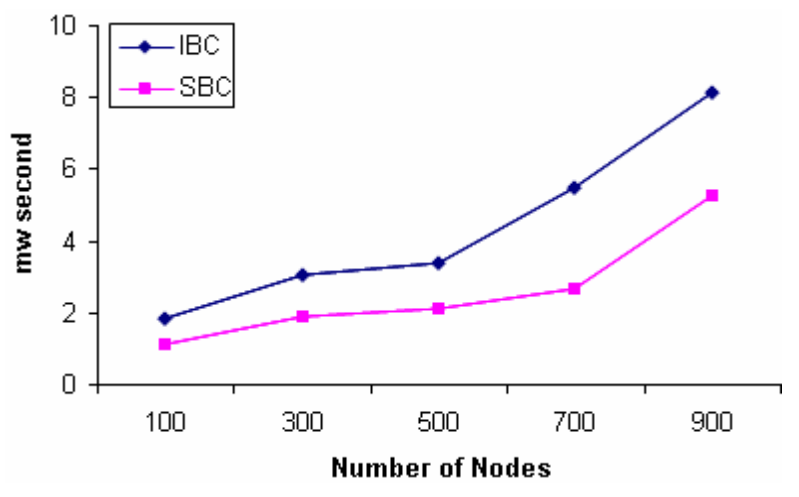

Fig. 5. Average power consumption per node in critical positions

\section{Conclusion}

In this paper, we had presented an agent strategy to allow the agent implemented in the sensor nodes to cleverly manage the power consumption of the sensor nodes. This optimizes the cooperation of agents in wireless sensor networks. This optimization is based on allowing the agent to take into consideration multiple parameters existing in its local view. In addition, to the available sensor node battery, this strategy deals with the position of the sensor node within the WSN and specially the critical position of a node (when a node connects two parts of the network). It takes into consideration the density of the network around the sensor node and the information importance degree. This strategy allows computing the relevance of a cooperation of an agent with other agents.

The importance of this strategy has been studied comparing to IBC through several simulations. The results illustrate the performance of our strategy. Indeed, SBC has proved an important optimization in term of average power consumption per node and an important management of the power of nodes in critical positions.

As a future work, we think that a mathematical model could be interesting to study the possibility of using a variable cooperation relevance threshold. Then, this agent strategy will be studied in the case of multiple applications over the same physical WSN. We aim also to explore the possibility of using an agent based routing protocol.

\section{References}

1. Chen, M., Kwon, T., Leung, V.C.M., Yuan, Y.: Mobile Agent Based Wireless Sensor Networks. Journal of Computers 1, 14-21 (2006)

2. Ferber, J.: Multi-Agent System: An Introduction to Distributed Artificial Intelligence. Addison Wesley Longman, Harlow (1999) 
3. Gan, L., Liu, J., Jin, X.: Agent-Based, Energy Efficient Routing in Sensor Networks. In: AAMAS. ACM, USA (2004)

4. Li, Y., Xu, J., Zhao, B., Yang, G.: A New Mobile agent architecture for wireless Sensor Networks. In: Industrial Electronics and Applications, ICIEA, pp. 1562-1565. IEEE, Los Alamitos (2008)

5. Liu, H., Liu, C.: An Intelligent Agent Routing over Wireless Sensor Networks. In: IEEE Proceedings of MASS 2006, pp. 358-366. IEEE, Los Alamitos (2006)

6. Marsh, D., O'Hare, G.M.P., Ruzzelli, A., Tynan, R.: Agents for Wireless Sensor Network Power Management. In: ICPP Workshops, pp. 413-418. IEEE Computer Society, Los Alamitos (2005)

7. Sardouk, A., Merghem_Boulahia, L., Gaiti, D.: Agents Cooperation for Power-Efficient Information Processing in Wireless Sensor Networks. In: Networking and Electronic Commerce Research Conference, Italy (2008)

8. Sardouk, A., Merghem_Boulahia, L., Gaiti, D.: Agent-Cooperation Based Communication Architecture for Wireless Sensor Network. IFIP Wireless Days/Ad-hoc and Wireless Sensor Networks. EAU (2008)

9. Sohraby, K., Minoli, D., Znati, T.: Wireless Sensor Networks, Technology, Protocols, and Applications. Wiley-Interscience, Hoboken (2007)

10. Sun Microsystem, Sun Labs: Sun ${ }^{\mathrm{TM}}$ Small Programmable Object Technology (Sun SPOT) Theory of Operation (2008)

11. U.P.C. Laboratory and W.A.M. Laboratory: Glomosim: A scalable simulation environment for wireless and wired network systems. In: The 3rd International Working Conference on Performance Modeling and Evaluation of Heterogeneous Networks (2005) 